\title{
New limit for mildly relativistic magnetic monopoles obtained with IceCube
}

\author{
The IceCube Collaboration ${ }^{\dagger}$ \\ ${ }^{\dagger}$ http://icecube.wisc.edu/collaboration/authors/icrc15_icecube \\ E-mail: obertackeeuni-wuppertal.de
}

\begin{abstract}
The existence of magnetic monopoles is a generic prediction of Grand Unified Theories (GUTs). Produced shortly after the Big Bang, these particles carry magnetic charge. Depending on the GUT model, the predicted monopole mass varies from $10^{7} \mathrm{GeV} / c^{2}$ to $10^{17} \mathrm{GeV} / c^{2}$. Magnetic monopoles could be accelerated to relativistic velocities by intergalactic magnetic fields. Due to these kinetic energies in combination with a moderate energy loss, they can pass through massive objects, such as the Earth, and remain relativistic.

Analogous to an electrical charge, a monopole traveling through IceCube with a velocity above $0.76 c$ produces direct Cherenkov light. Above $\sim 0.4 c$, also indirect Cherenkov light is produced via $\delta$-electrons knocked off by the monopole. The Cherenkov light can be detected by the IceCube sensors.

The analysis presented here is distinct from previous searches for relativistic and non-relativistic magnetic monopoles performed by IceCube as it focuses on the intermediate velocity region, $0.4 c<\beta<0.76 c$, using indirect Cherenkov light. We describe the method used in this search, and present the best limit to date in this velocity region, using one year of IceCube data from 2011.
\end{abstract}

Corresponding author: A. Obertacke Pollmann ${ }^{* 1}$

${ }^{1}$ Dept. of Physics, University of Wuppertal, 42119 Wuppertal, Germany

The 34th International Cosmic Ray Conference,

30 July- 6 August, 2015

The Hague, The Netherlands

${ }^{*}$ Speaker. 


\section{Introduction}

Magnetic monopoles are particles carrying an isolated magnetic charge, a multiple of the Dirac charge [1] $g=\frac{e}{2 \alpha} \approx 68.5 e$ where $e$ is the elementary electric charge and $\alpha$ is the fine structure constant. Magnetic monopoles are generic solutions of Grand Unified Theories (GUT) $[2,3]$. In this context they would be created in the GUT phase of the universe shortly after the Big Bang. They are one of the most promising candidates for physics beyond the standard model. GUT theories predict particle masses reaching from $10^{7} \mathrm{GeV} / c^{2}$ to $10^{17} \mathrm{GeV} / c^{2}$. Magnetic monopoles can be accelerated to relativistic velocities in intergalactic magnetic fields.

Water or ice neutrino telescopes, such as IceCube, instrument a large volume and are therefore able to detect rare events. This analysis searches for light signals from magnetic monopoles in the IceCube data recorded from May 2011 to May 2012.

IceCube is a cubic-kilometer neutrino detector installed in the ice at the geographic South Pole [4] between depths of $1450 \mathrm{~m}$ and $2450 \mathrm{~m}$. Detector construction started in 2005 and was completed in 2010. Neutrino reconstruction relies on the optical detection of Cherenkov radiation emitted by secondary particles produced in neutrino interactions in the surrounding ice or the nearby bedrock.

Magnetic monopoles faster than the Cherenkov threshold in ice, $v_{C} \approx 0.76 c$, emit Cherenkov light. In addition monopoles ionize the surrounding matter, and most of the knocked-off $\delta$-electrons are ejected with energies sufficient to produce Cherenkov light. This mechanism is called indirect Cherenkov light emission and produces light down to a monopole velocity of $\sim 0.4 c$. The yield of direct and indirect Cherenkov light along a monopole track is shown in Fig. 1.

Monopoles were simulated in a velocity range of $0.4 c \leq v_{M} \leq 0.995 c$. The simulation of monopoles uses the standard IceCube software adjusted to generate and propagate monopoles as well as generating and propagating the Cherenkov light that they emit. For further details, see [5]. The characteristic signature of a (mildly) relativistic monopole in IceCube is a through-going track with very high, but constant brightness. An event view of the signal from a monopole simulated in IceCube is shown in Fig. 2.

\section{Event selection}

The most abundant background type in this analysis are muons or muon bundles produced in cosmic ray air showers. Since they traverse the detector from above, but monopoles are expected to come from every direction. A cut on the reconstructed zenith angle of a recorded IceCube event removes most of the background while keeping a substantial portion of the signal. When two or more air showers hit the detector at the same time, a time/space clustering algorithm is used to separate them into different events. This improves reconstruction quality by rejecting most of the coincident events, however there still remain many coincident events left to be discarded with other methods, for example with a cut on the length of the longest track segment without any hits closer than $100 \mathrm{~m}$.

A boosted decision tree (BDT) is trained to remove the remaining background. To focus on low monopole velocities $\leq v_{C}$ and improve the final sensitivity in this parameter range, just this kind of signal simulation is used to train the BDT. 


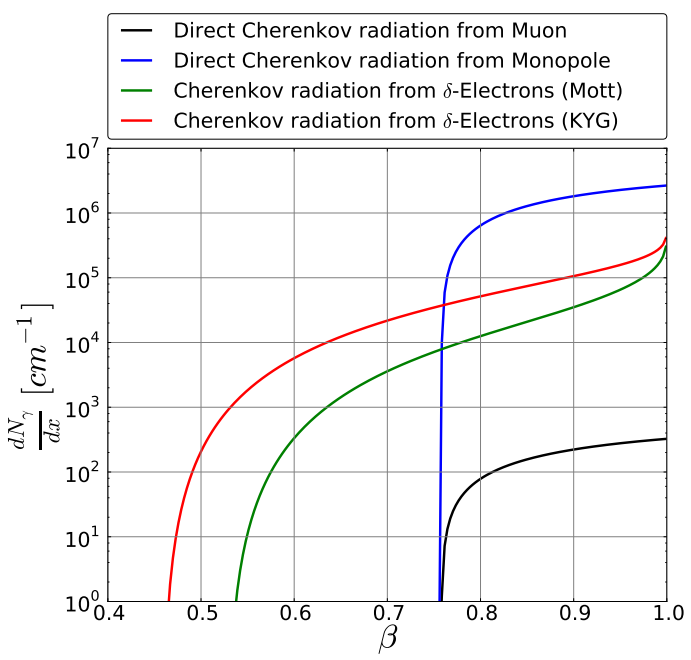

Figure 1: The number of photons per $\mathrm{cm}$ produced by a bare relativistic monopole (blue) is 4700 times higher than the light yield of a bare muon (black) producing direct Cherenkov light.

The light yield of all $\delta$-electrons calculated using the KYG cross section [6] (red) is used in this analysis in comparison to the simple Mott cross section [7] (green). Above the Cherenkov threshold indirect and direct light are summed up in simulation.

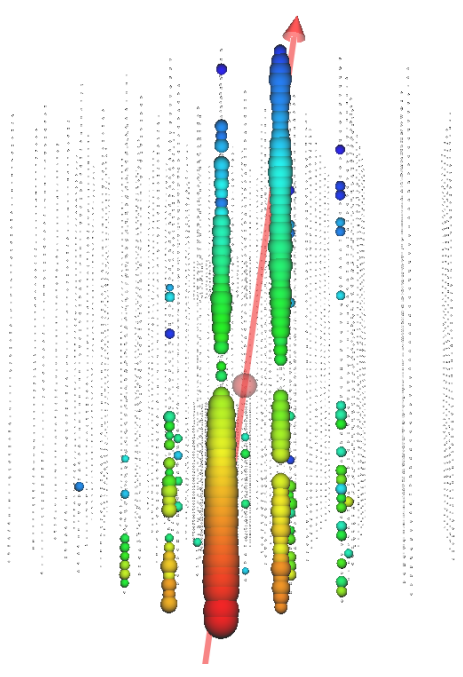

Figure 2: A view of a simulated magnetic monopole event with a velocity of $0.83 c$ and a zenith angle of $\sim 170$ degrees, using both direct and indirect Cherenkov light. The positions of IceCube DOMs are shown with gray dots, hit DOMs with colored spheres. Their size is scaled with the number of recorded photons. The color denotes the time development from red to blue. The red line shows the reconstructed track.

After all cuts, including the BDT cut, the remaining background are charged particles produced in interactions of atmospheric neutrinos. The energies reach from $3.3 \cdot 10^{2} \mathrm{GeV}$ to $2.6 \cdot 10^{4} \mathrm{GeV}$.

IceCube analyses are performed in a blind way to avoid bias. This is done by using only simulated background and signal. To validate the simulations, $10 \%$ of the recorded data are used. However, at this point there was not sufficient background simulation available. Therefore pullvalidation was used, which is a re-sampling method inspired by bootstrapping. This method enables the estimation of the uncertainties of the background rate at low-statistics and is described in detail in [8]. Finally, the BDT cut, shown in Fig. 3, in its re-sampled distribution was optimized using a modified Feldman-Cousin approach which also considerates uncertainties [9].

The velocity distribution of background after the last cut, estimated with pull-validation, is shown in Fig. 4. The total uncertainties of this analysis are also shown. The detector simulation gives an uncertainty of $3.0 \%$ on the monopole rate. The theoretical flux model is taken into account for the uncertainty of the neutrino flux in addition to the detector simulation which gives $16.9 \%$ of total systematic uncertainty. The variation in the background rate estimate obtained with resampled BDTs was used as an additional, largest source of uncertainty.

This results in a background rate of 0.55 events with a $90 \%$ confidence upper limit of 3.61 events. 


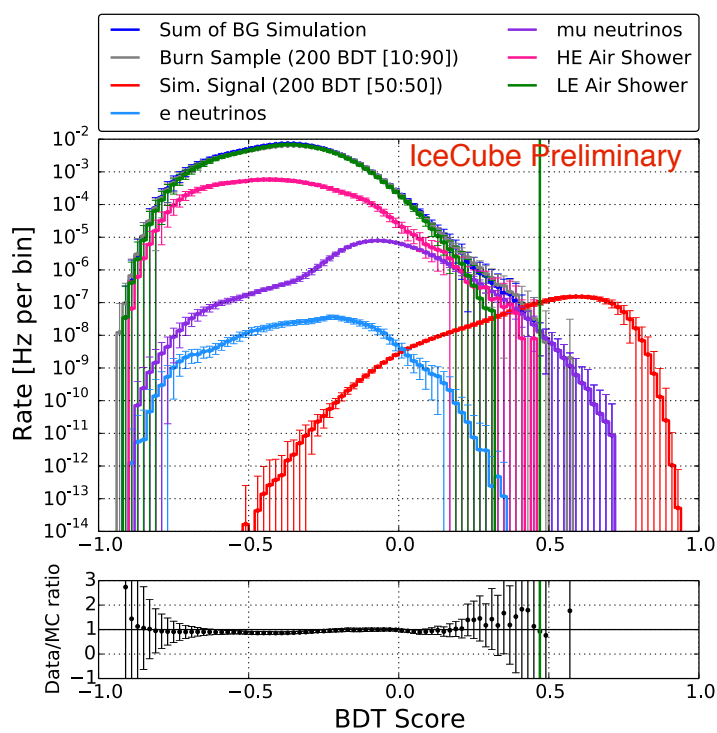

Figure 3: 200 BDTs are trained on a random subsample of $10 \%$ of IceCube data. The mean and standard deviation per bin is shown and compared with different types of background simulation. In the signal region atmospheric neutrinos dominate the background contribution.

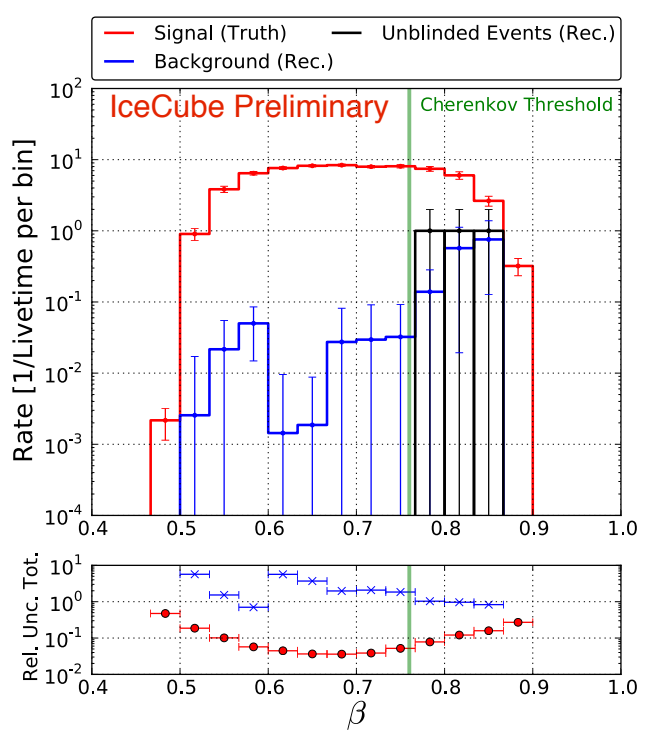

Figure 4: Velocity distribution used to calculate the final differential limit. Reconstructed velocity is used for background and true simulated velocity for signal. The lower part of the plot shows the velocity dependence of the total uncertainties including resampling.

\section{Result}

After the analysis procedure was finalized and all selection criteria fixed, the remaining $90 \%$ of available data from the IceCube season 2011/2012 was used to derive the result. Three events with velocities $\geq v_{C}$ survived the event selection. This is consistent with background. Fig. 4 shows these events in comparison to the expectation.

Two events have a clear background signature, when inspected visually, because they stop in the detector. One event traverses the whole detector but has light output fainter than expected from a monopole of this velocity. All three events are therefore treated as an upward fluctuation of the background weakening the limit.

The limit for different velocities at the detector is shown in Fig. 6. It extends from $0.51 c$ to $0.877 c$ and for most of this range improves previous limits by a factor of almost two orders of magnitude. For a more detailed description of this analysis see [5]. 


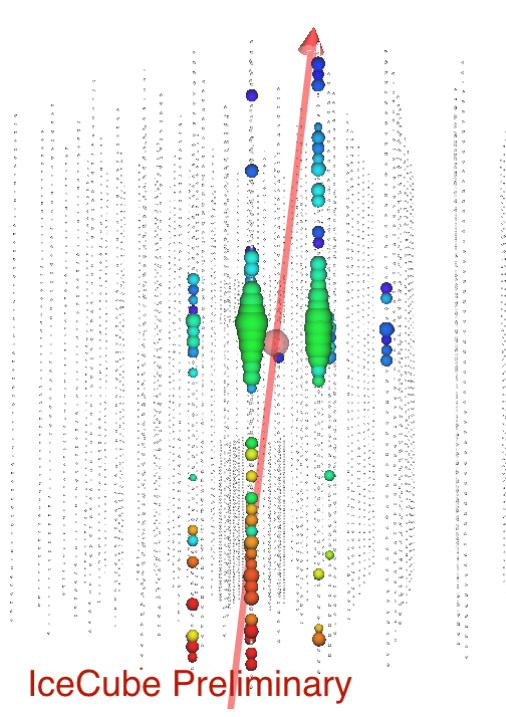

Figure 5: One of three events which passed all cuts in this analysis with a BDT Score of 0.53. 110 optical sensors are hit as opposed to an expectation of $196 \pm 11$ for a monopole with the velocity and direction as shown in Fig. 2.

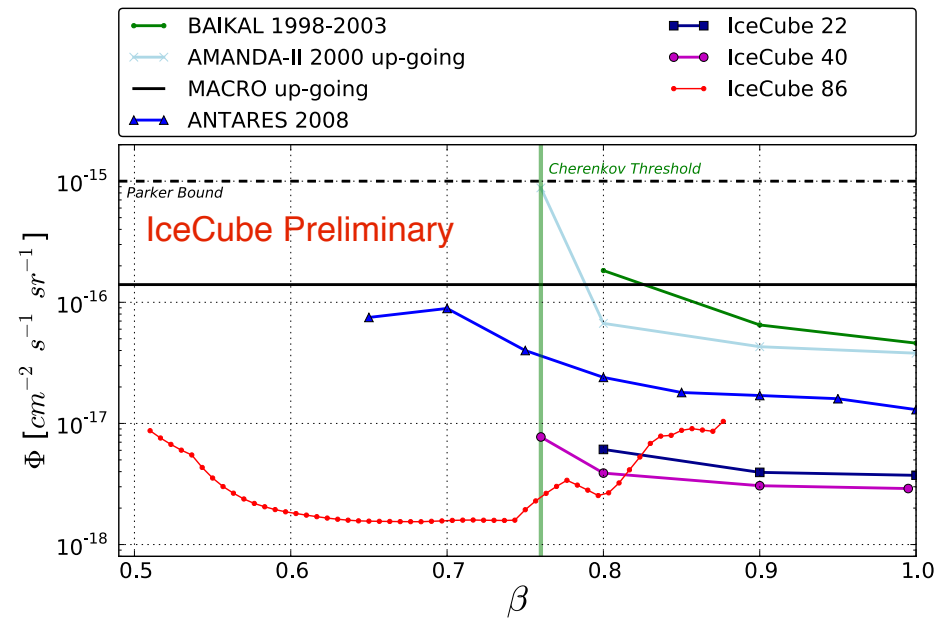

Figure 6: Limit of this analysis (red) compared to other analyses. The lines of all limits are only drawn to guide the eyes. This analysis compares directly with limits from ANTARES [10] and MACRO [11]. Other limits are from BAIKAL [12], AMANDA [13], and IceCube in its 22 [14] and 40 [15] string configuration.

\section{References}

[1] P. Dirac, Proc. Roy. Soc. A 133 (1931) 60-73.

[2] G. 't Hooft, Nucl. Phys. B 79 (1974) 276-284.

[3] A. M. Polyakov, JETP Lett. 20 (1974) 194-195.

[4] IceCube Collaboration, A. Achterberg, et al., Astropart. Phys. 26 (2006) 155-173.

[5] A. Obertacke Pollmann, $\mathrm{PhD}$ thesis, University of Wuppertal. In Preparation.

[6] Y. Kazama, C. N. Yang, and A. S. Goldhaber, Phys. Rev. D 15 (1977) 2287-2299.

[7] S. P. Ahlen, Phys. Rev. D 14 (1975) 2935-2940.

[8] IceCube Collaboration, in these proceedings, no. PoS(ICRC2015)1211.

[9] G. J. Feldman and R. D. Cousins, Phys. Rev. D 57 (1998) 3873-3889.

[10] ANTARES Collaboration, S. Adrián-Martínez, et al., Astropart. Phys. 35 (2012) 634-640.

[11] MACRO Collaboration, M. Ambrosio, et al., Eur. Phys. J. C 25 (2002) 511-522.

[12] BAIKAL Collaboration, V. Aynutdinov, et al., Astrophys. J. 29 (2008) 366-372.

[13] IceCube Collaboration, R. Abbasi, et al., Eur. Phys. J. C 69 (2010) 361-378.

[14] IceCube Collaboration, R. Abbasi, et al., Phys. Rev. D 87 (2013) 022001 1-11.

[15] J. Posselt, PhD thesis, University of Wuppertal, 2013. 